\title{
QUASI-STATIC DEFORMATION DUE TO A DISLOCATION SOURCE IN A MAXWELLIAN VISCOELASTIC EARTH MODEL
}

\author{
Takaya IWASAKI* \\ Geophysical Institute, Faculty of Science, \\ the University of Tokyo, Tokyo, Japan \\ (Received August 6, 1984; Revised January 8, 1985)
}

In this paper, we treat quasi-static deformation field due to a dislocation source in a composite medium which consists of elastic layers overlying a stratified viscoelastic half-space. The rheologies of the viscoelastic layers are assumed to be elastic dilatational and Maxwell deviatoric. Integral representations of the surface displacements are derived from those in the associated elastic problem by applying the correspondence principle of linear viscoelasticity. The effect of gravity is taken into account by modifying the boundary conditions with respect to normal stress.

Features of the viscoelastic displacements are investigated for a threelayered structure model composed of an elastic surface layer, an intervening low viscosity layer, and a viscoelastic substratum with relatively high viscosity. A dislocation source is supposed to be located in the elastic surface layer. The effect of the stress relaxation in the substratum is examined for both a point source and a dimensional fault. Difference in the viscosity of the substratum affects the amount and extent of the viscoelastic deformation. In a model with a low viscous substratum, the amount of displacement is large and the deformation spreads in a broad area. The effect of gravity is investigated assuming a pure dip-slip fault. The viscoelastic deformation for the gravitating case is restrained in amount and extent as compared with the non-gravitating case. Such an effect of gravity becomes notable with time. In the gravitating case, the stress relaxation progresses fast, and the apparent relaxation time of deformation is much shorter than that for the non-gravitating case.

\section{Introduction}

Transient crustal movement following a large earthquake is one of the interesting phenomena concerned with anelastic properties of the earth. Such a postseismic crustal motion is considered to be controlled by quasi-static stress relaxation in a viscoelastic region existing in the upper part of the earth.

The earth's crust and upper mantle are characterized by three layers with different rheology, namely, an elastic surface layer (lithosphere), an intervening low

* Present address: Laboratory for Ocean Bottom Seismology, Faculty of Science, Hokkaido University, Sapporo, Japan 
viscosity layer (asthenosphere), and a substratum with relatively high viscosity. From analyses of postglacial uplifts, the viscosity and thickness of the low viscosity layer have been determined to be $10^{20}-10^{21}$ poises and $100-200 \mathrm{~km}$, respectively (MCConell, 1968; Walcott, 1973; CATHLES, 1975; IWASAKI and Matsu'URA, 1982). The viscosity of the substratum has been estimated as about $10^{22}$ poises, which is greater than that of the asthenosphere by one or two orders.

Thus far, several authors have made theoretical investigation on quasi-static deformation due to faulting using a multi-layered earth model. NuR and MAvko (1974) treated quasi-static displacements for a two-layered model under plane-strain approximation, where an earthquake is represented as a screw or an edge dislocation in an elastic surface layer overlying a substratum of standard linear solid. Within a similar framework, CoHEN (1979) investigated surface deformations due to a screw dislocation in a surface layer of standard linear solid overlying an elastic dilatational and Maxwell deviatoric half-space. Such problems have been extended to a three-dimensional case by RUNDLE and JACKSON (1977) and RUNDLE (1978), assuming the rheological property of the half-space to be Maxwell deviatoric, but not to be elastic dilatational. COHEN $(1980 \mathrm{a}, 1980 \mathrm{~b})$ treated quasi-static displacements, strains, and stresses caused by a strike-slip fault in a stratified half-space composed of a surface layer of standard linear solid and Maxwellian viscoelastic substratum. The gravity effect on the deformation due to a dislocation source was examined for a two-layered model with a Maxwellian viscoelastic half-space by RUNDLE (1982). In the above-mentioned investigations, viscoelastic ductile region is assumed to extend infinitely under a brittle surface layer. Such modeling is too simple in comparison with the actual rheological structure of the earth's crust and upper mantle.

MATSU'URA et al. (1981) and IWASAKI and MATSU'URA (1981) investigated quasi-static displacements, strains, and tilts caused by faulting in a stratified elastic half-space with an intervening Maxwellian viscoelastic layer. This model, however, seems to be inadequate in treating crustal deformations with a relatively long time scale, because the stress relaxation under the low viscosity layer is not taken into account and the gravity effect is neglected.

In the present study, the crust and upper mantle of the earth are modeled by a stratified medium composed of elastic layers and an intervening viscoelastic layer overlying a viscoelastic substratum with relatively high viscosity. Considering mechanical properties of the earth, the viscoelastic layers are supposed to be elastic dilatational and Maxwell deviatoric. In Section 2, we present expressions of viscoelastic deformations due to a point dislocation source, extending the mathematical treatments by MATSU'URA et al. (1981). The effect of gravity is introduced by modifying the boundary conditions with respect to normal stress. In Section 3 , we present several representative numerical examples, and investigate features of viscoelastic deformation fields caused by faulting. 


\section{Mathematical Formulation}

Consider a stratified half-space which consists of $n$-parallel, homogeneous, and isotropic layers overlying a substratum, where the $n$-th layer and the substratum are supposed to be viscoelastic (elastic dilatational and Maxwell deviatoric)

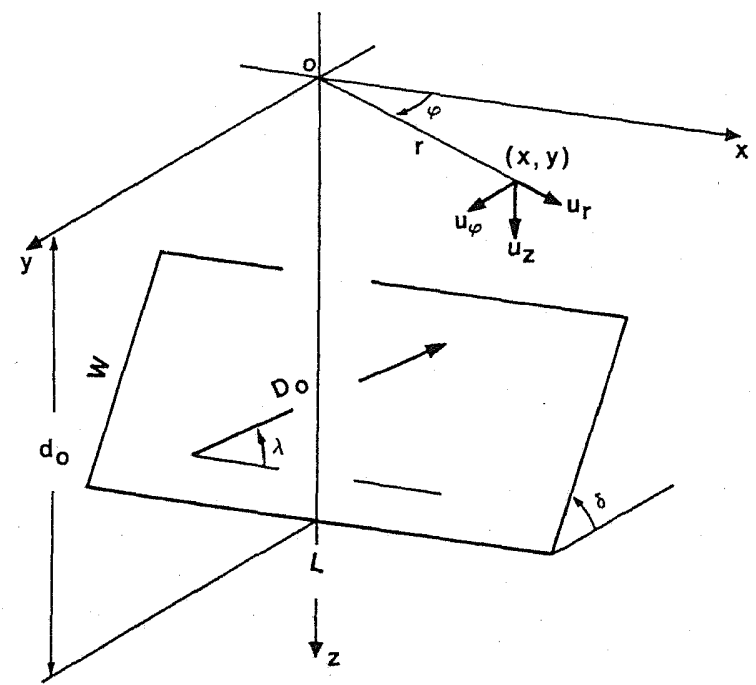

(a)

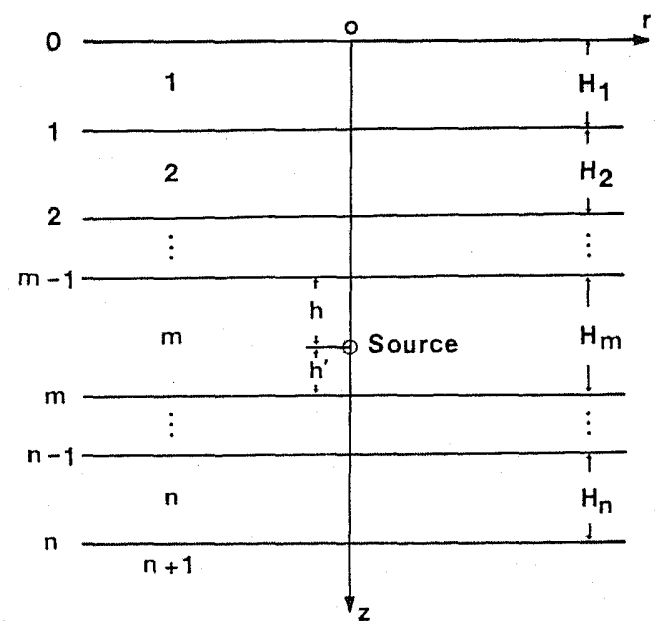

(b)

Fig. 1. Coordinates, geometries, and notations. (a) Geometry of the dislocation source model. $D_{0}$, final dislocation; $\delta$, dip-angle; $\lambda$, slip-angle; $L$, fault length; $W$, fault width; $d_{\mathrm{o}}$, depth of the lower fault margin, and $u_{r(\varphi, z)}$, displacement components in the cylindrical coordinates. (b) Numbering of layers and interfaces. $H_{i}$, thickness of the $i$-th layer. 
and others perfectly elastic. Denoting Lamè elastic constants of the $k$-th layer by $\lambda_{k}$ and $\mu_{k}(k=1, \ldots, n+1)$, a stress-strain relation for each medium is given as follows; for the elastic layer,

$$
\sigma_{i j}=\lambda_{k} \varepsilon_{m m} \delta_{i j}+2 \mu_{k} \varepsilon_{i j} \quad(k \neq n, n+1),
$$

and for the viscoelastic layer with viscosity, $\eta_{k}$,

$$
\dot{\sigma}_{i j}+\frac{\mu_{k}}{\eta_{k}}\left(\sigma_{i j}-\frac{1}{3} \sigma_{m m} \delta_{i j}\right)=\lambda_{k} \dot{\varepsilon}_{m m} \delta_{i j}+2 \mu_{k} \dot{\varepsilon}_{i j} \quad(k=n, n+1),
$$

where $\sigma_{i j}, \varepsilon_{i j}$, and $\delta_{i j}$ are the stress tensor, strain tensor, and the unit diagonal tensor, respectively, and the dot means differentiation with respect to time.

In the previous paper (MATSU'URA et al., 1981), which is referred to as Paper I, we developed a method to compute quasi-static deformations due to a dislocation source in a stratified elastic half-space with an intervening viscoelastic layer by applying a well-known correspondence principle of linear viscoelasticity. IWASAKI and MATSU'URA (1982) obtained expressions of displacement field due to an axially symmetric load applied on the surface of a composite medium which consists of elastic layers and stratified viscoelastic half-space. Hereafter, this paper is referred to as Paper II. Mathematical treatments in the present study are quite similar to those in Papers I and II. The coordinates, geometries, and notations used in the following descriptions are given in Fig. 1.

Suppose a case in which a point dislocation source with a time function of a step type is located in the $m$-th layer of the composite medium and on the $z$-axis at a depth $h$ from the upper boundary of the source existing layer.

The effect of gravity is introduced approximately by modifying boundary conditions in the non-gravitating case (MCConell, 1965). Denoting the gravity acceleration by $g$, the displacement components in the $k$-th layer by $w_{r}^{k}, w_{\varphi}^{k}$, and $w_{z}^{k}$, and the stress components by $\sigma_{z r}^{k}, \sigma_{z \varphi}^{k}$, and $\sigma_{z z}^{k}$, the modified boundary conditions at the $k$-th interface are given by

$$
\left.\begin{array}{lll}
w_{r}^{k}=w_{r}^{k+1}, & w_{\varphi}^{k}=w_{\varphi}^{k+1}, & w_{z}^{k}=w_{z}^{k+1}, \\
\sigma_{z r}^{k}=\sigma_{z r}^{k+1}, & \sigma_{z \varphi}^{k}=\sigma_{z \varphi}^{k+1}, & \sigma_{z z}^{k}-\rho_{k} g w_{z}^{k}=\sigma_{z z}^{k+1}-\rho_{k+1} g w_{z}^{k+1},
\end{array}\right\}
$$

where $\rho_{k}$ is the density of the $k$-th layer. At the ground surface, we impose the following conditions,

$$
\sigma_{z r}^{1}=0, \quad \sigma_{z \varphi}^{1}=0, \quad \sigma_{z z}^{1}-\rho_{1} g w_{z}^{1}=0 .
$$

As is shown in Paper II, the gravity effect at the $k$-th interface is smaller than that at the ground surface by one order. Hence, as far as we treat crustal deformations with relatively small wavelength $\left(\leq 10^{3} \mathrm{~km}\right)$, the gravity effect at the interface is negligible.

According to the mathematical procedures described in Papers I and II, the quasi-static displacement, $w_{r(\varphi, z)}$, is represented as a sum of an instantaneous 
elastic response at the time of faulting and a transient viscoelastic movement following the event, namely,

$$
w_{r(\varphi, z)}(r, \varphi, t)=u_{r(\varphi, z)}(r, \varphi, t)+v_{r(\varphi, z)}(r, \varphi, t),
$$

where $u_{r(\varphi, z)}$ and $v_{r(\varphi, z)}$ are the elastic and viscoelastic parts of the displacement field, respectively. The expressions of $u_{r(\varphi, z)}$ for the non-gravitating case are given by Eq. (5) in Paper I. In the gravitating case, $u_{r(\varphi, z)}$ has the same form as that for the non-gravitating case except that the $4 \times 4$ matrix, $P$, defined by Eq. (12) in Paper I is replaced with $\boldsymbol{P} \cdot \boldsymbol{G}_{0}$. Here, the matrix $\boldsymbol{G}_{0}$ represents the gravity effect at the ground surface, and its definition is given by Eq. (A.I-4) in Paper II. The expressions for $v_{r,(\varphi, z)}$, on the other hand, are obtained as follows;

$$
\begin{aligned}
v_{r}(r, \varphi, t) & \\
= & \frac{D_{\circ}}{4 \pi} H(t) \sum_{l=0}^{2}\left[K_{l}(\varphi) \int_{0}^{\infty}\left\{V_{r, 1}(t, \xi) \mathrm{e}^{-h \xi}+(-1)^{l} V_{r, 2}(t, \xi) \mathrm{e}^{-h^{\prime} \xi}\right\} \frac{\partial J_{l}(\xi r)}{\partial r} \mathrm{~d} \xi\right. \\
& -L_{l}(\varphi) \int_{0}^{\infty}\left\{V_{r, 3}(t, \xi) \mathrm{e}^{-h \xi}+(-1)^{l} V_{r, 4}(t, \xi) \mathrm{e}^{-h^{\prime} \xi}\right\} \frac{\partial J_{l}(\xi r)}{\partial r} \mathrm{~d} \xi \\
& \left.+\alpha_{m} h^{\prime} L_{l}(\varphi) \int_{0}^{\infty} \xi\left\{V_{r, 1}(t, \xi) \mathrm{e}^{-h \xi}-(-1)^{l} V_{r, 2}(t, \xi) \mathrm{e}^{-h^{\prime} \xi}\right\} \frac{\partial J_{l}(\xi r)}{\partial r} \mathrm{~d} \xi\right] \\
& -\frac{D_{0}}{4 \pi} H(t) \sum_{l=1}^{2} \frac{\partial^{2} L_{l}(\varphi)}{\partial \varphi^{2}} \int_{0}^{\infty}\left\{V_{\varphi, 1}(t, \xi) \mathrm{e}^{-h \xi}-(-1)^{l} V_{\varphi, 2}(t, \xi) \mathrm{e}^{-h^{\prime} \xi}\right\} \frac{J_{l}(\xi r)}{r} \mathrm{~d} \xi, \\
v_{\varphi}(r, & \varphi, t) \\
= & \frac{D_{0}}{4 \pi} H(t) \sum_{l=1}^{2}\left[\frac{\partial K_{l}(\varphi)}{\partial \varphi} \int_{0}^{\infty}\left\{V_{r, 1}(t, \xi) \mathrm{e}^{-h \xi}+(-1)^{l} V_{r, 2}(t, \xi) \mathrm{e}^{-h^{\prime} \xi}\right\} \frac{J_{l}(\xi r)}{r} \mathrm{~d} \xi\right. \\
& -\frac{\partial L_{l}(\varphi)}{\partial \varphi} \int_{0}^{\infty}\left\{V_{r, 3}(t, \xi) \mathrm{e}^{-h \xi}+(-1)^{l} V_{r, 4}(t, \xi) \mathrm{e}^{-h^{\prime} \xi}\right\} \frac{J_{l}(\xi r)}{r} \mathrm{~d} \xi \\
& \left.+\alpha_{m} h^{\prime} \frac{\partial L_{l}(\varphi)}{\partial \varphi} \int_{0}^{\infty} \xi\left\{V_{r, 1}(t, \xi) \mathrm{e}^{-h \xi}-(-1)^{l} V_{r, 2}(t, \xi) \mathrm{e}^{-h^{\prime} \xi}\right\} \frac{J_{l}(\xi r)}{r} \mathrm{~d} \xi\right] \\
& +\frac{D_{0}}{4 \pi} H(t) \sum_{l=1}^{2} \frac{\partial L_{l}(\varphi)}{\partial \varphi} \int_{0}^{\infty}\left\{V_{\varphi, 1}(t, \xi) \mathrm{e}^{-h \xi}-(-1)^{l} V_{\varphi, 2}(t, \xi) \mathrm{e}^{-h^{\prime} \xi}\right\} \frac{\partial J_{l}(\xi r)}{\partial r} \mathrm{~d} \xi, \\
v_{z}(r, & \varphi, t) \\
= & \frac{D_{\circ}}{4 \pi} H(t) \sum_{l=0}^{2}\left[K_{l}(\varphi) \int_{0}^{\infty} \xi\left\{V_{z, 1}(t, \xi)^{-h \xi}+(-1)^{l} V_{z, 2}(t, \xi) \mathrm{e}^{-h^{\prime} \xi}\right\} J_{l}(\xi r) \mathrm{d} \xi\right. \\
& -L_{l}(\varphi) \int_{0}^{\infty} \xi\left\{V_{z, 3}(t, \xi) \mathrm{e}^{-h \xi}+(-1)^{l} V_{z, 4}(t, \xi) \mathrm{e}^{-h^{\prime} \xi \xi}\right\} J_{l}(\xi r) \mathrm{d} \xi \\
& \left.+\alpha_{m} h^{\prime} L_{l}(\varphi) \int_{0}^{\infty} \xi^{2}\left\{V_{z, 1}(t, \xi) \mathrm{e}^{-h \xi}-(-1)^{l} V_{z, 2}(t, \xi) \mathrm{e}^{-h^{\prime} \xi}\right\} J_{l}(\xi r) \mathrm{d} \xi\right] . \\
&
\end{aligned}
$$

with

$$
\alpha_{m}=\left(\lambda_{m}+\mu_{m}\right) /\left(\lambda_{m}+2 \mu_{m}\right), \quad h^{\prime}=H_{m}-h,
$$


and

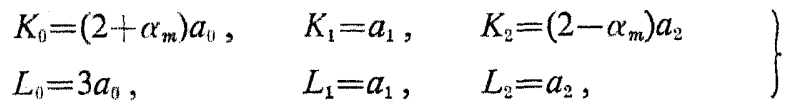

where $D_{\circ}$ is the final dislocation, $H(t)$ the Heaviside step function, $J_{l}(\xi r)$ the Bessel function of oder $l, H_{m}$ the thickness of the $m$-th layer, $h$ the depth of the dislocation source from the $m-1$ interface, and $a_{l}(l=0,1,2)$ are functions of dip-angle $(\delta)$, slip-angle $(\lambda)$, and azimuth $(\varphi)$ which are defined by Eq. (8) in Paper I.

$V_{r(\varphi, z), j}$ in Eq. (6), which are called kernel functions, represent the viscoelastic effect of layering. Taking a time constant by

$$
\tau=\eta_{n} / \mu_{n},
$$

$V_{r(\varphi, z), j}$ are expressed as

$$
\begin{gathered}
V_{r(z), j}(t, \xi)=-\sum_{i=1}^{7} \frac{C_{r(z), j}^{(i)}(\xi)}{\zeta_{i}(\xi)}\left(1-\mathrm{e}^{\tau_{i(\xi) t / \tau}}\right), \\
V_{\varphi, j}(t, \xi)=-\sum_{i=1}^{2} \frac{C_{\varphi, j}^{(i)}(\xi)}{\zeta_{i}^{\prime}(\xi)}\left(1-\mathrm{e}^{\zeta_{i}^{\prime}(\xi) t / \tau}\right),
\end{gathered}
$$

where $\zeta_{i}(\xi)$ and $\zeta_{i}^{\prime}(\xi)$ are real negative and coefficients, $C_{r(z), j}^{(i)}(\xi)$ and $C_{\varphi, j}^{(i)}(\xi)$, are defined in Appendix. It should be noted that the viscoelastic part vanishes at $t=0$, and tends to a final steady state at $t=\infty$.

Evaluation of integrals in Eq. (6) is carried out by using a technique developed in Paper I. As an example, we consider a case of a three-layered model given by Table 1, in which a point dislocation source is located in the elastic surface layer. Figure 2 shows the several kernel functions, $V_{r(\varphi, z), j}$ at $t=10 \tau$ where $\tau$ (unit of time) and $H$ (unit of length) are taken to be 4.81 years and $100 \mathrm{~km}$, respectively. These kernel functions can be approximated as follows;

$$
\bar{V}_{r(\varphi, z), j}(t, \xi)=\sum_{i=1}^{n} a_{r(\varphi, z), j}^{(i)}(t) \xi^{b(t)}(\dot{t}, z), j^{(t)} \mathrm{e}^{-c_{r(\varphi, z), j(t) \hat{\xi}}^{(t)}},
$$

with

$$
b_{r(\varphi, z), j}^{(i)}(t) \geq 0, \quad c_{r(\varphi, z), j}^{(i)}(t) \geq 0 .
$$

The number of terms, $n$, in Eq. (11) is taken as seven in the present study.

Table 1. Structure model adopted for the computation of kernel functions.

\begin{tabular}{cccccc}
\hline No. & $H(\mathrm{~km})$ & $V_{\mathrm{P}}(\mathrm{km} / \mathrm{s})$ & $V_{\mathrm{S}}(\mathrm{km} / \mathrm{s})$ & $\rho\left(\mathrm{g} / \mathrm{cm}^{3}\right)$ & $\eta$ (poises) \\
\hline 1 & 60.0 & 7.8 & 4.5 & 3.2 & $\infty$ \\
2 & 120.0 & 8.2 & 4.4 & 3.4 & $10^{20}$ \\
3 & - & 8.7 & 4.7 & 3.6 & $10^{21}$ \\
\hline
\end{tabular}



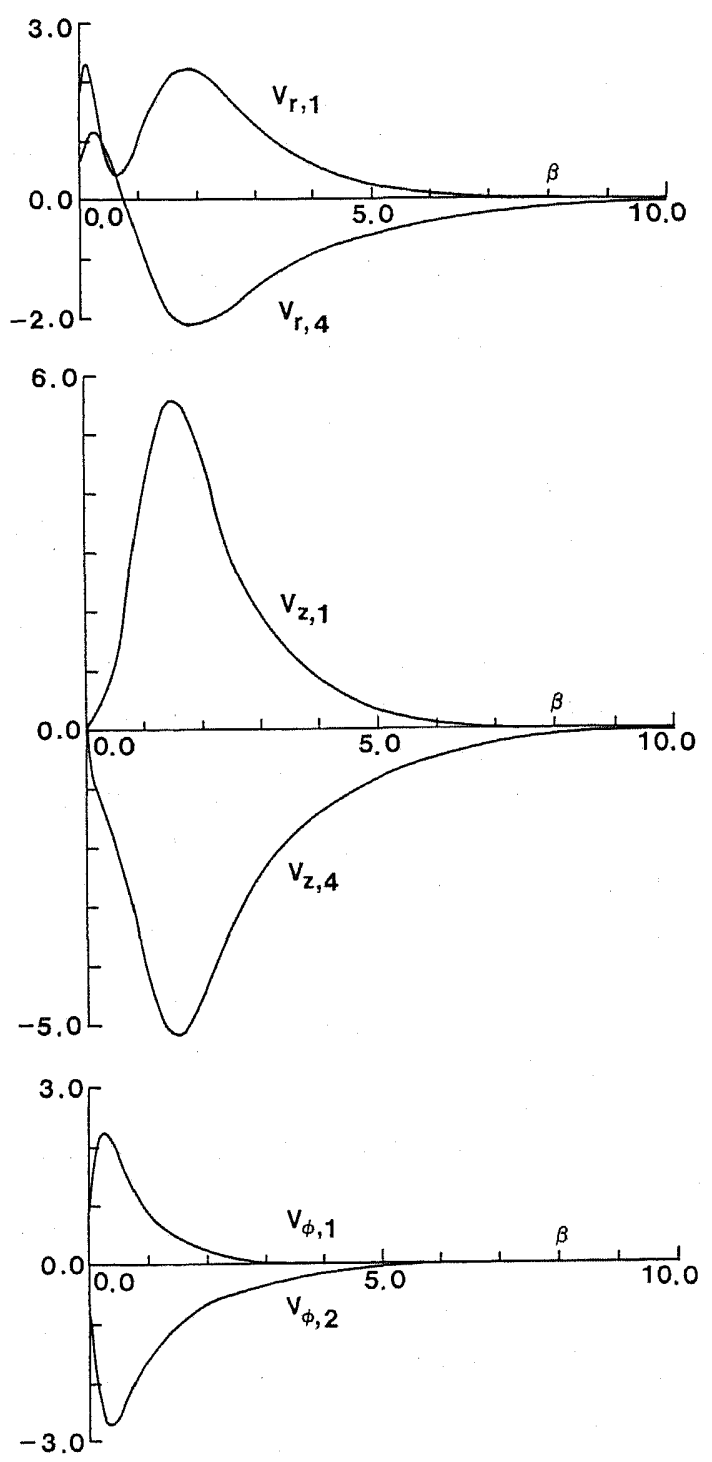

Fig. 2. Several kernel functions. For the gravitating three-layered model in Table 1 , the values of $V_{r(\varphi, z), j}$ at the time of $10 \tau$ are shown as a function of $\beta(=\xi H)$. The unit of time ( $\tau)$ and unit of length $(H)$ are taken as 4.81 years and $100 \mathrm{~km}$, respectively.

Substituting the approximate kernel functions $\bar{V}_{r(\varphi, z), j}$ into Eq. (6) instead of $V_{r(\varphi, z), j}$, the viscoelastic part of the quasi-static displacements can be evaluated as a sum of the Gauss' hypergeometric functions.

The quasi-static displacements due to a dimensional fault are obtained by numerical integration of the solutions for a point source over the fault plane. Usually, this process requires a rather long computation time. In the present case, a technique for saving the computation time, which was developed by SATO and MATSU'URA (1973), is available. 


\section{Numerical Examples}

This section presents several numerical examples of quasi-static displacements due to a dislocation source. The structure model adopted for the computation is of a three-layered type as is given in Table 2. It should be noted that this model contains two extreme cases: (i) if $H_{\mathrm{v}}=\infty$, the model represents a two-layered model composed of an elastic surface layer overlying a viscoelastic substratum, and (ii) if $\eta^{\prime}=\infty$, a three-layered model with an elastic substratum. In each case of the following examples, we take unit of time $\left(\tau=\eta / \mu_{2}\right)$ and unit of length $(H)$ as 4.81 years and $100 \mathrm{~km}$, respectively.

\subsection{Effect of stress relaxation in the viscoelastic substratum}

In order to investigate the stress relaxation effect of the viscoelastic substratum, we present numerical examples computed for representative three-layered models with different viscosity ratio $\left(\eta^{\prime} / \eta\right)$. In Paper I, we clarified features of quasistatic deformations in two extreme cases of $\eta^{\prime} / \eta=1$ (two-layered model) and $\eta^{\prime} / \eta=\infty$ (three-layered model with an elastic substratum). For comparison with the previous results, we consider the non-gravitating case.

First, we examine the effect of the difference in viscosity ratio on kernel functions. For three cases of $\eta^{\prime} / \eta=2,10$, and $100, V_{r, 2}$ and $V_{z, 4}$ at various times are shown as a function of $\beta(=\xi H)$ in Fig. 3. Here, the thickness of the intervening layer $\left(H_{\mathrm{v}}\right)$ is fixed at $60 \mathrm{~km}$. Figures 2 and 4 in Paper I are also referred to as the cases of $\eta^{\prime} / \eta=\infty$ and 1 , respectively.

From comparison of these figures, it is found that the difference in viscosity ratio affects notably the behavior of kernel functions at small $\beta$. In the case of $\eta^{\prime} / \eta=2$, the kernel functions near $\beta=0$ increase rapidly with time as compared with the cases of $\eta^{\prime} / \eta=10$ and 100 . As the viscosity of the substratum increases, the growth rate of the kernel function becomes small. In the extreme case of $\eta^{\prime} / \eta=\infty$, the value of the kernel function at $\beta=0$ tends to zero (see Fig. 2 in Paper I).

Next, we investigate variations of displacement profiles with time. For this purpose, it is convenient to take the Cartesian coordinate system $(x, y, z)$ shown in

Table 2. Structure model adopted for the computation of numerical examples.

\begin{tabular}{cccccc}
\hline No. & $H(\mathrm{~km})$ & $V_{\mathrm{P}}(\mathrm{km} / \mathrm{s})$ & $V_{\mathrm{S}}(\mathrm{km} / \mathrm{s})$ & $\rho\left(\mathrm{g} / \mathrm{cm}^{3}\right)$ & $\eta$ (poises) \\
\hline 1 & 60.0 & 7.8 & 4.5 & 3.2 & $\infty$ \\
2 & $H_{\mathrm{v}}$ & 8.2 & 4.4 & 3.4 & $\eta\left(=10^{20}\right)$ \\
3 & - & 8.7 & 4.7 & 3.6 & $\eta^{\prime}$ \\
\hline
\end{tabular}

Fig. 3. Representative kernel functions for the three-layered models with different viscosity ratio. The kernel functions at various times are presented for the models with $\eta^{\prime} / \eta=2,10$, and 100 . In each case, the thickness of the intervening viscoelastic layer $\left(H_{\mathrm{v}}\right)$ is taken to be $60 \mathrm{~km}$. (a) $\eta^{\prime} / \eta=2$. (b) $\eta^{\prime} / \eta=10$. (c) $\eta^{\prime} / \eta=100$. 

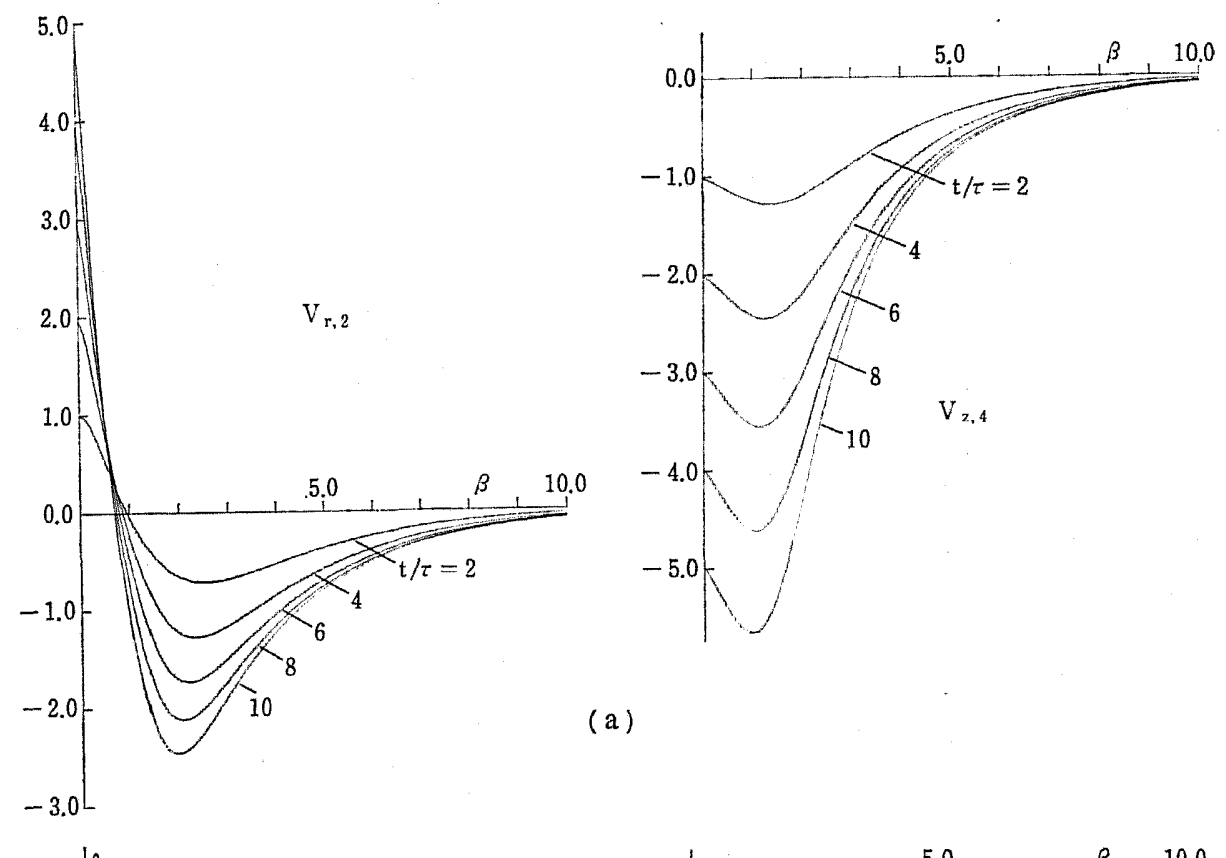

(a)
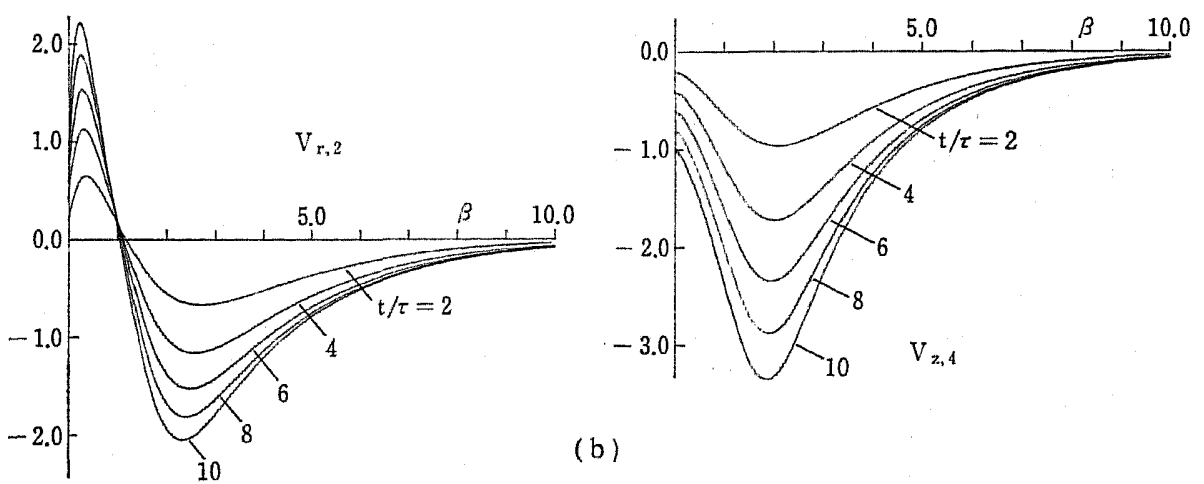

(b)
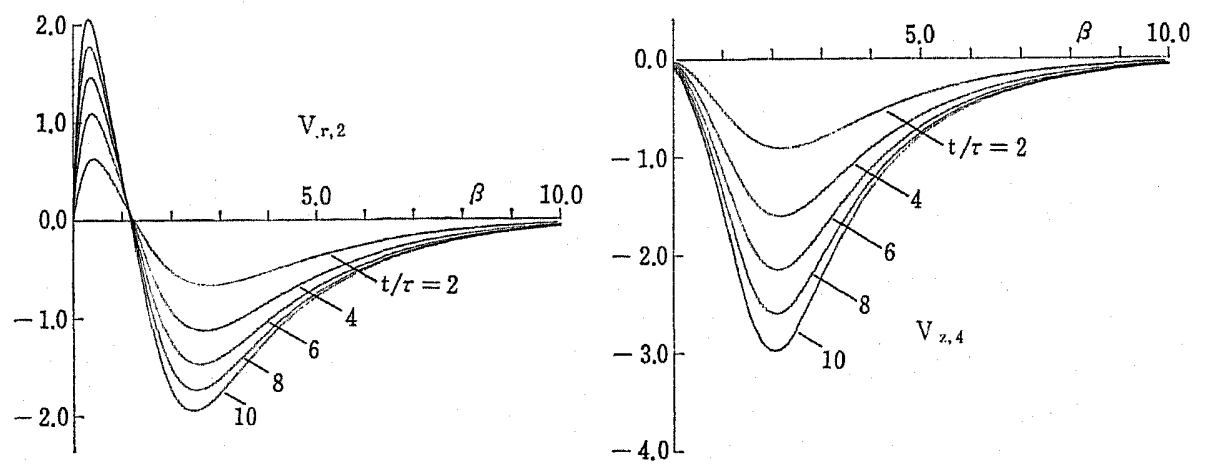

(c)

Fig. 3 


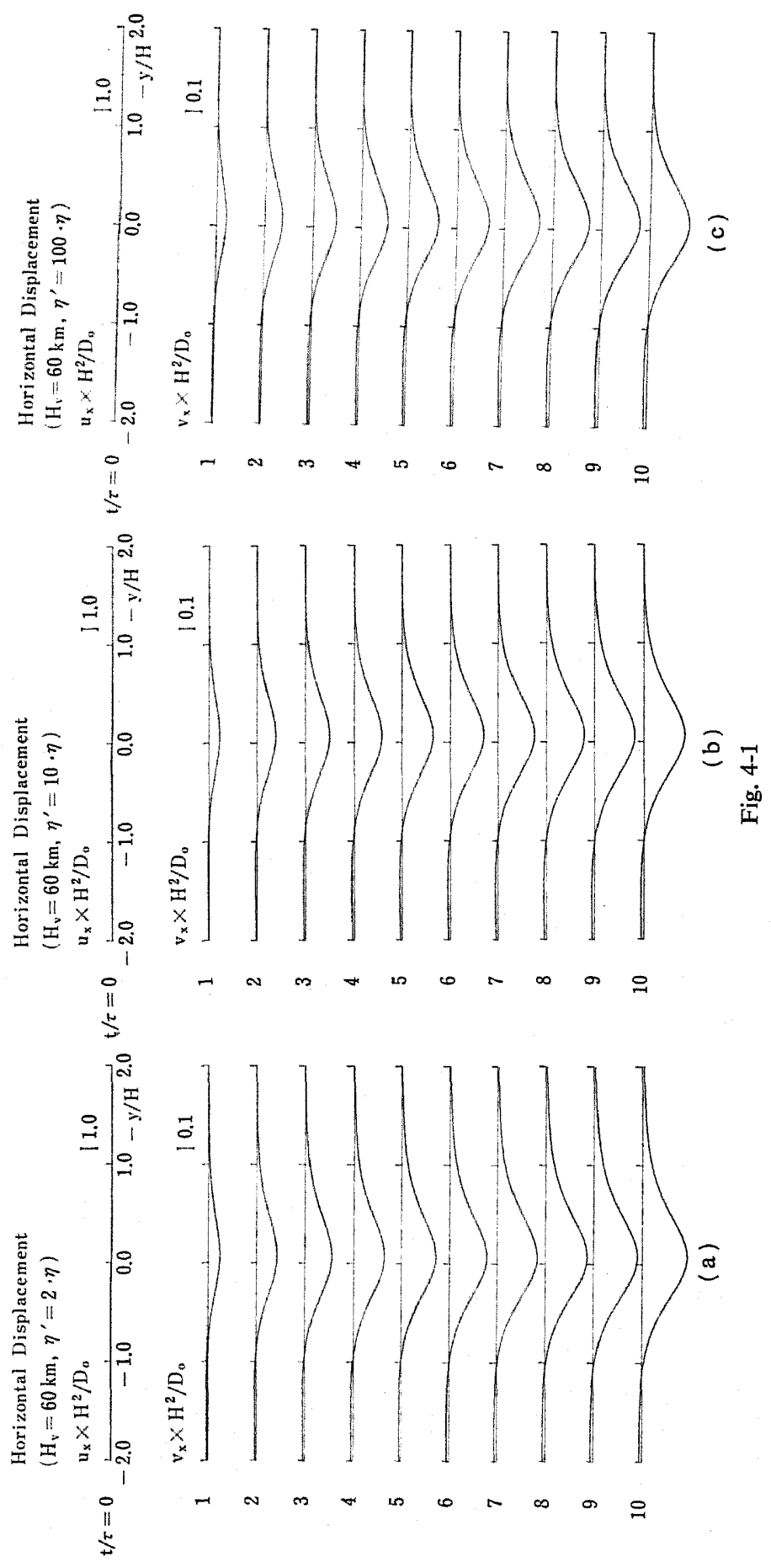




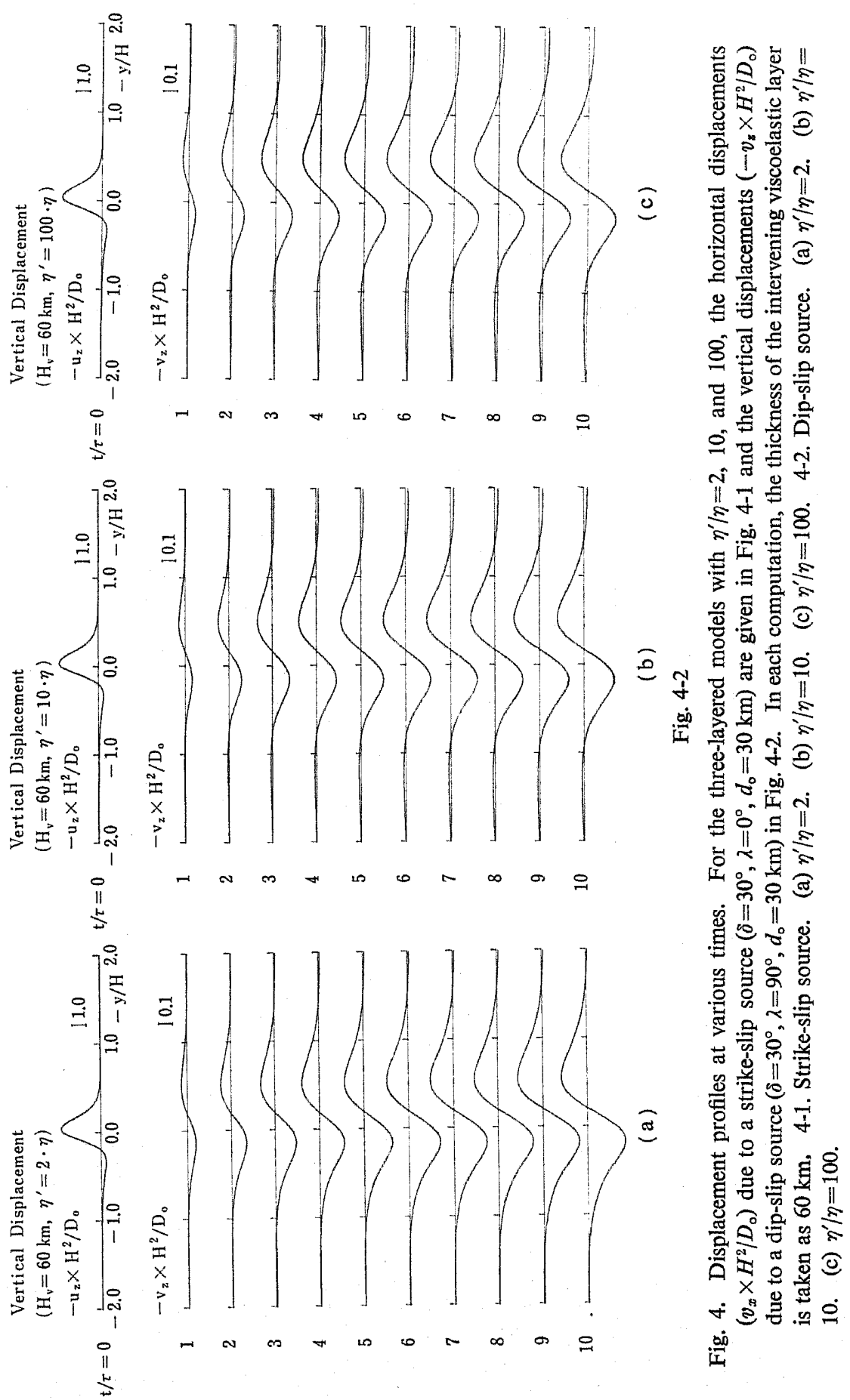




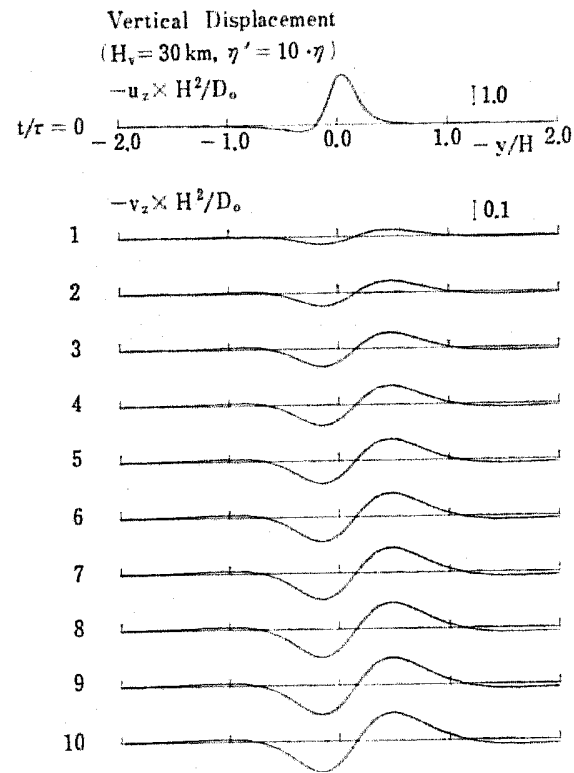

(a)

Fig. 5-1

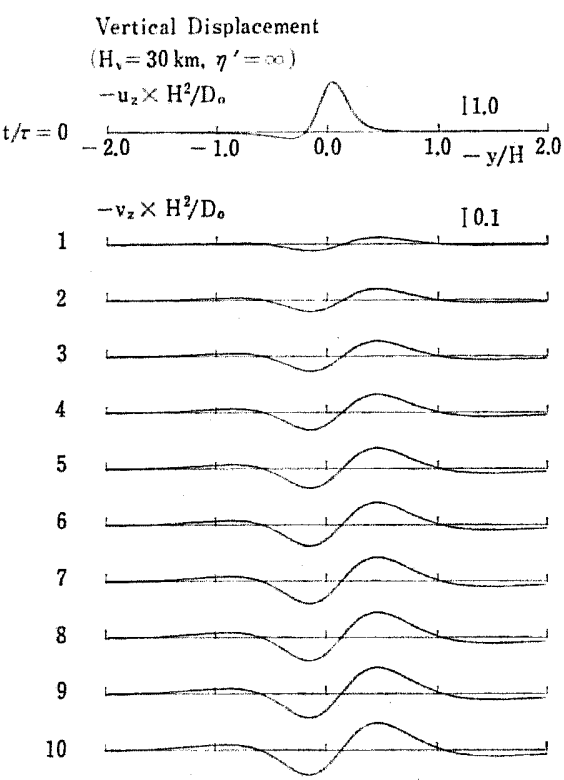

(b)

Vertical Displacement

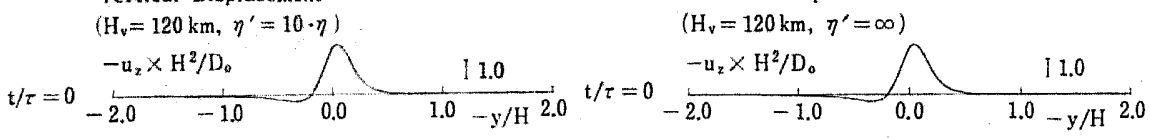

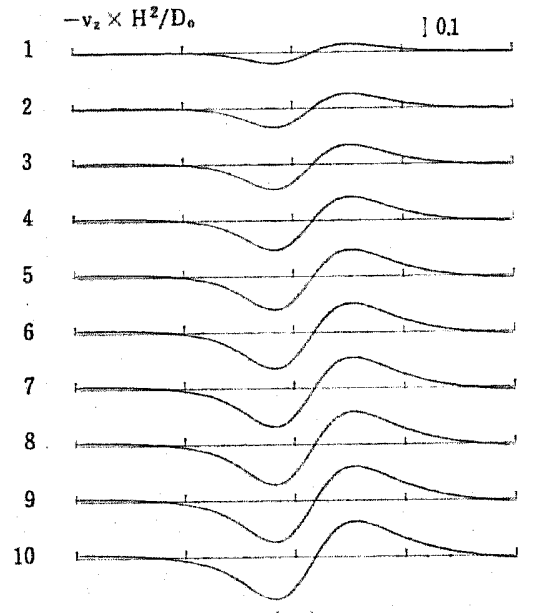

(a)

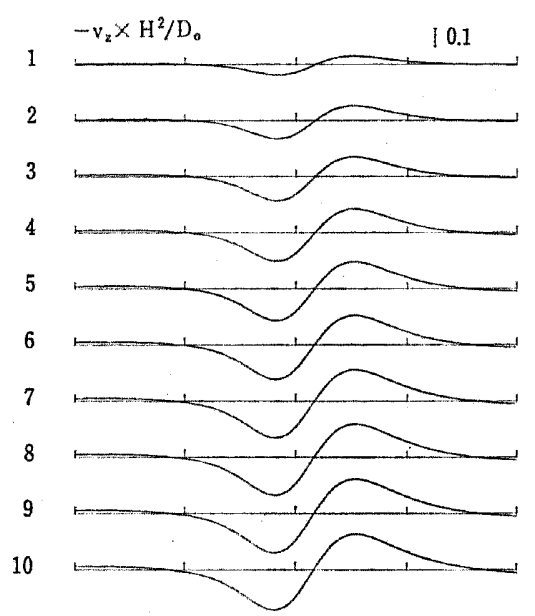

(b)

Fig. 5-2

Fig. 5. Displacement profiles at various times. Taking the thickness of the intervening viscoelastic layer as $30 \mathrm{~km}$ and $120 \mathrm{~km}$, the displacement profiles due to a dip-slip source are computed for cases of $\eta^{\prime} / \eta=10$ and $\infty$. The source parameters are taken to be the same as those in Fig. 4-2. 5-1. Three-layered model with $H_{\mathrm{r}}=$ $30 \mathrm{~km}$. (a) $\eta^{\prime} / \eta=10$. (b) $\eta^{\prime} / \eta=\infty$. 5-2. Three-layered model with $H_{\mathrm{v}}=120 \mathrm{~km}$. (a) $\eta^{\prime} / \eta=10$. (b) $\eta^{\prime} / \eta=\infty$. 
Fig. 1. Displacement components in this system are related with those in the cylindrical coordinates by

$$
\left[\begin{array}{l}
w_{x} \\
w_{y} \\
w_{z}
\end{array}\right]=\left[\begin{array}{ccc}
\cos \varphi & -\sin \varphi & 0 \\
\sin \varphi & \cos \varphi & 0 \\
0 & 0 & 1
\end{array}\right]\left[\begin{array}{l}
w_{r} \\
w_{\varphi} \\
w_{z}
\end{array}\right],
$$

with

$$
r=\left(x^{2}+y^{2}\right)^{1 / 2}, \quad \cos \varphi=x / r, \quad \sin \varphi=y / r .
$$

Let us consider a case in which a point dislocation source with a dip angle $(\delta)$ of $30^{\circ}$ is located at a depth $\left(d_{0}\right)$ of $30 \mathrm{~km}$ in the elastic surface layer of the three-layered model.

We take the thickness of the intervening layer $\left(H_{\mathrm{v}}\right)$ to be $60 \mathrm{~km}$. For the three cases of $\eta^{\prime} / \eta=2,10$, and 100 , horizontal displacements $\left(v_{x} \times H^{2} / D_{0}\right)$ on the $y$ axis due to a strike-slip source $\left(\lambda=0^{\circ}\right)$ are shown in Fig. 4-1, and vertical displacements $\left(-v_{z} \times H^{2} / D_{0}\right)$ due to a dip-slip source $\left(\lambda=90^{\circ}\right)$ in Fig. 4-2. The elastic part is given in the uppermost part of each figure, where the scale of the elastic part is taken to be $1 / 10$ of that for the viscoelastic part. The displacement profiles for two extreme cases of $\eta^{\prime} / \eta=1$ and $\infty$ are presented in Figs. 8 and 7 of Paper I, respectively. As is seen in these figures, the amplitude of the viscoelastic displacement increases with time, and the deformed area extends out slowly from the source. The displacement profiles for $\eta^{\prime} / \eta=10$ and 100 are quite similar to the

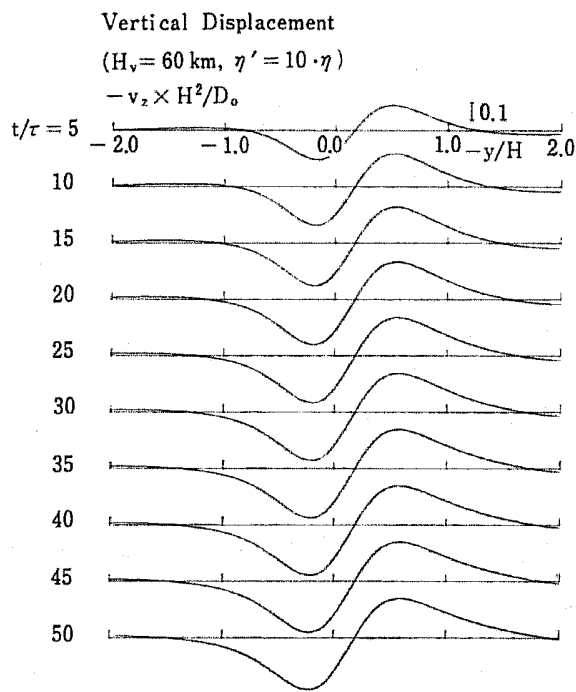

(a)

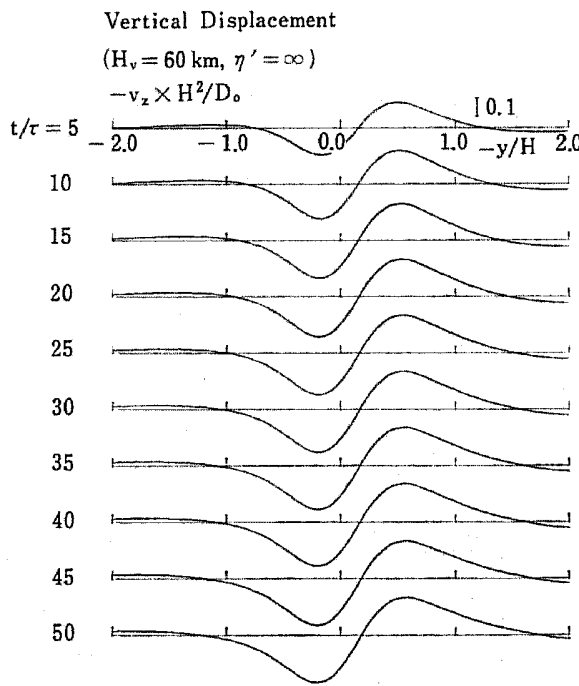

(b)

Fig. 6. Displacement profiles at various times. Vertical displacements due to a dipslip source are computed up to the time of $50 \tau$ for the three-layered model with $H_{\nabla}=$ $60 \mathrm{~km}$. The source parameters are taken to be the same as those in Fig. 5. (a) $\eta^{\prime} / \eta$ $=10$. (b) $\eta^{\prime} / \eta=\infty$. 

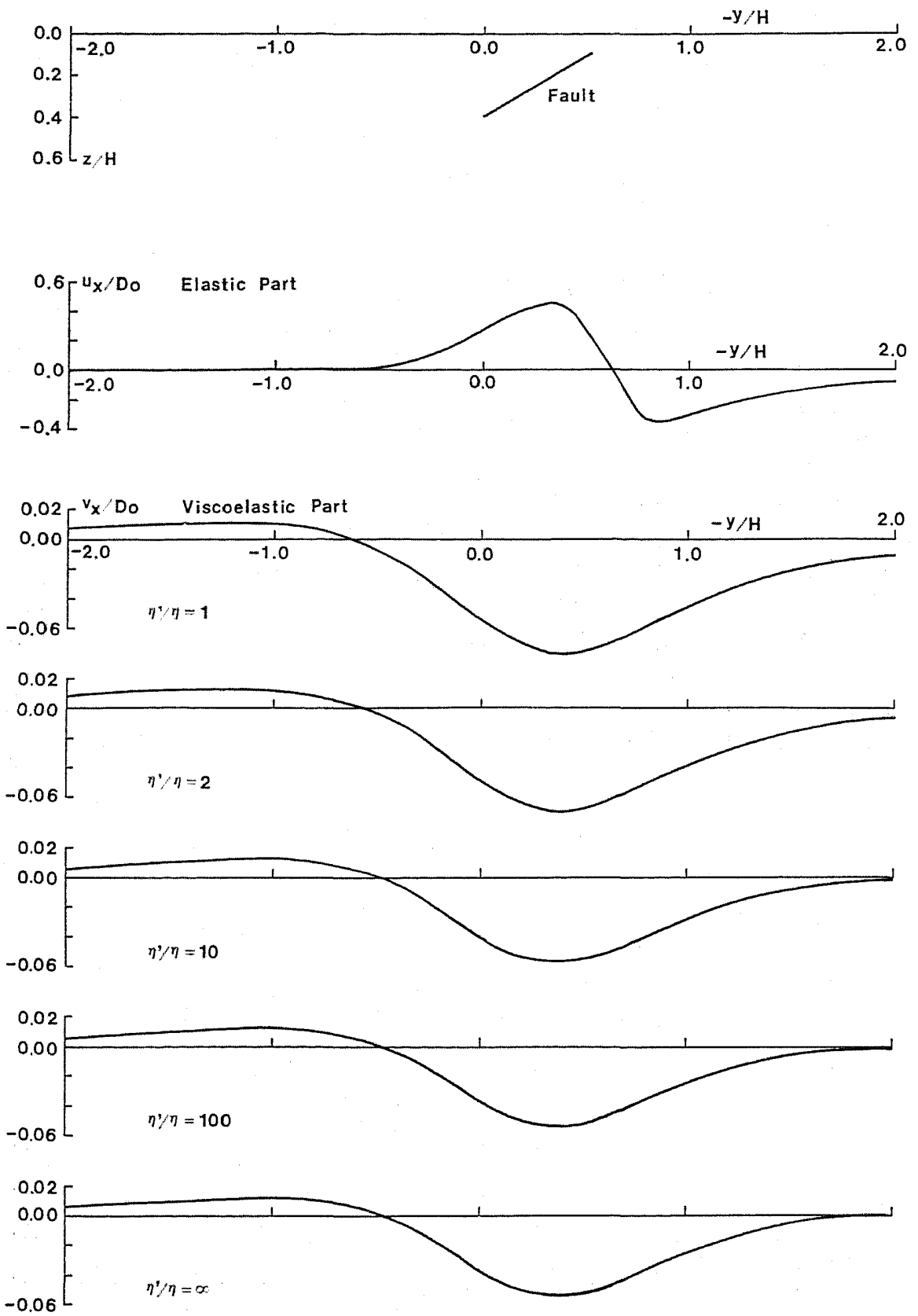

Fig. 7(a)

Fig. 7. Profiles of quasi-static displacements due to a dimensional fault with $\delta=30^{\circ}$, $L=120 \mathrm{~km}, W=60 \mathrm{~km}$, and $d_{0}=40 \mathrm{~km}$. For the three-layered models with different viscosity ratio $\left(\eta^{\prime} / \eta=1,2,10,100\right.$, and $\left.\infty\right)$, the horizontal displacements due to the pure strike-slip fault $\left(\lambda=0^{\circ}\right)$ are presented in Fig. 7(a) and the vertical displace- 

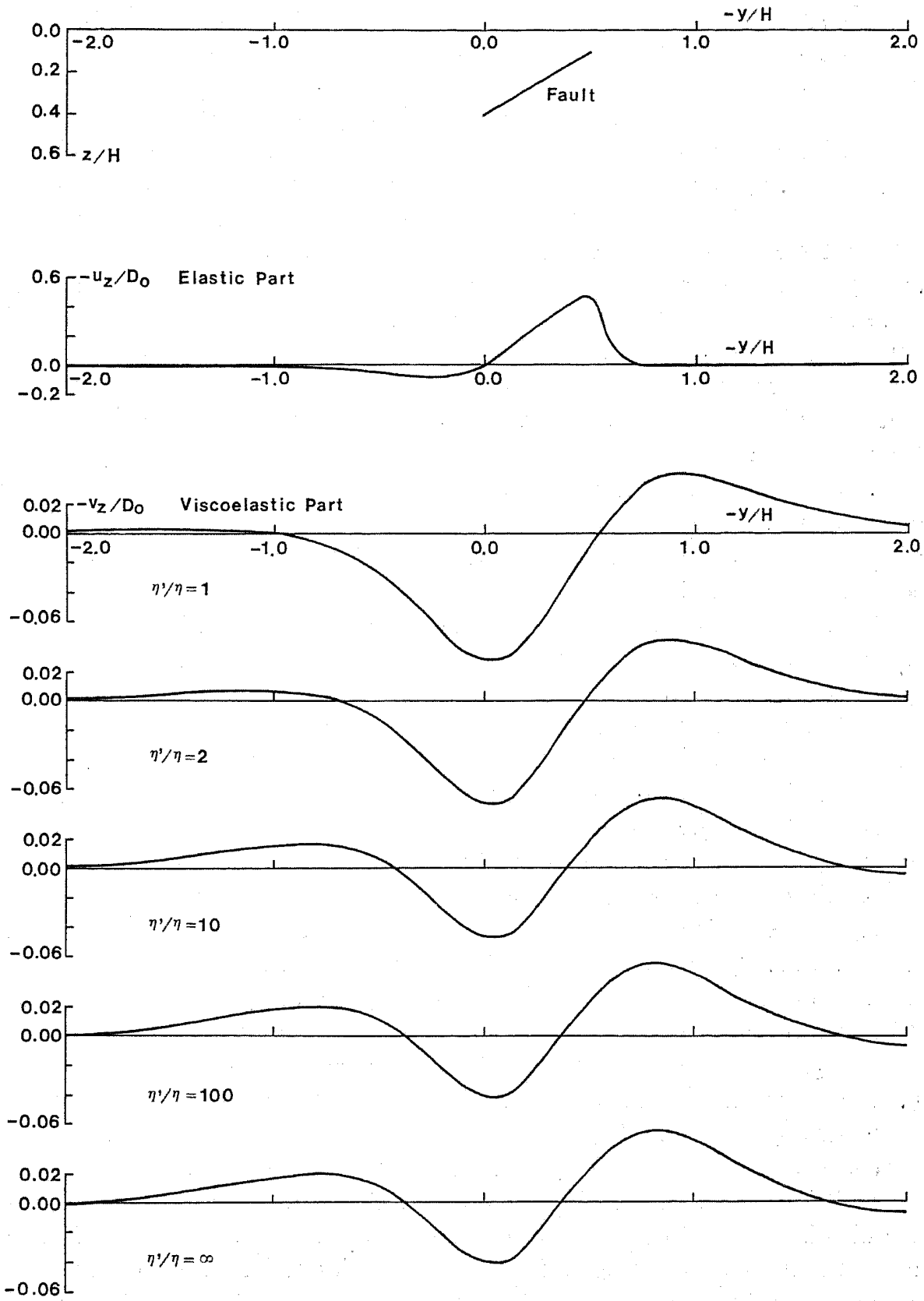

Fig. 7(b)

ments due to the pure dip-slip fault $\left(\lambda=90^{\circ}\right)$ in Fig. $7(\mathrm{~b})$. The viscoelastic part is computed at the time of $5 \tau$. In each computation, the thickness of the intervening layer $\left(H_{\mathrm{v}}\right)$ is fixed at $60 \mathrm{~km}$. 
case of $\eta^{\prime} \eta \eta=\infty$. This indicates that the stress relaxation in the substratum progresses very slowly in these two cases and its effect on the surface deformation is small. In the case of $\eta^{\prime} / \eta=2$, the amplitude of viscoelastic displacement is 1.10 1.25 times greater than that for $\eta^{\prime} / y_{1}=10$, and the predominant wavelength of the deformation is considerably long. Such differences in displacement profile are to be expected from the behavior of the kernel functions described above.

The effect of the stress relaxation in the substratum depends on the thickness of the intervening layer $\left(H_{\mathrm{v}}\right)$. We examine two cases of $H_{\mathrm{v}}=30$ and $120 \mathrm{~km}$, assuming a pure dip-slip source with $\delta=30^{\circ}$ located at a depth of $30 \mathrm{~km}$. In Fig. 5, the vertical displacements for $\eta^{\prime} / \eta=10$ are compared with those for $\eta^{\prime} / \eta=\infty$. It should be noted that the stress relaxation in the model with $\eta^{\prime} / \eta=\infty$ is restricted within the intervening viscoelastic layer. In the case of $H_{\mathrm{v}}=30 \mathrm{~km}$, the displacement profile in a region of $y / H \geq 0.6$ is affected by the difference in viscosity ratio. The maximum amplitude for $\eta^{\prime} / \eta=10$ is $25 \%$ greater than that for $\eta^{\prime} / \eta=\infty$. In the case of $H_{\mathrm{v}}=120 \mathrm{~km}$, on the other hand, the profiles for $\eta^{\prime} / \eta=10$ and $\infty$ are similar, and the difference in maximum amplitude is only about $5 \%$. In general, the stress relaxation effect of the substratum becomes obscure as the thickness of the intervening layer increases.

For the two cases of $\eta^{\prime} / \eta=10$ and $\infty$, long-term variations of vertical displacement are examined in Fig. 6 . In these examples, the thickness of the intervening layer is set to $60 \mathrm{~km}$, and the source parameters are taken to be the same as those in Fig. 4-2. The difference between the profiles for $\eta^{\prime} / \eta=10$ and $\infty$ becomes clear with increase of time. In the case of $\eta^{\prime} / \eta=10$, the deformation spreads in a broad area in comparison with the case of $\eta^{\prime} / \eta=\infty$. At $t=50 \tau$, the maximum displacement for $\eta^{\prime} / \eta=10$ is $10 \%$ greater than that for $\eta^{\prime} / \eta=\infty$. These results show that the stress relaxation effect of the substratum is not negligible in treating quasi-static crustal movements with a relatively long time scale.

Profiles of surface displacements due to a dimensional fault are investigated for the cases of $\eta^{\prime} / \eta=1,2,10,100$, and $\infty$, taking $H_{\mathrm{v}}$ as $60 \mathrm{~km}$. As the fault model, we assume a rectangular fault with $\delta$ (dip-angle) $=30^{\circ}, L$ (fault length) $=120 \mathrm{~km}$, $W$ (fault width) $=60 \mathrm{~km}$, and $d_{\circ}$ (depth of the lower fault margin) $=40 \mathrm{~km}$.

In Fig. 7 , we present horizontal displacements $\left(v_{x} / D_{\circ}\right)$ on the $y$-axis due to a pure strike-slip fault (Fig. 7(a)), and vertical displacements $\left(-v_{z} / D_{\mathrm{o}}\right)$ due to a pure dip-slip fault (Fig. 7(b)). The elastic part is shown in the upper part of each figure, where the scale is taken to be $1 / 10$ of that for the viscoelastic part. From these figures, it is found that the difference in viscosity ratio affects notably the viscoelastic displacement field. In the case of the strike-slip fault, the amplitude of the deformation strongly depends on the viscosity ratio. The maximum displacement for the case of $\eta^{\prime} / \eta=1$ is $7.7 \times 10^{-2}$ in unit of $D_{0}$, which is about 1.5 times greater than that for $\eta^{\prime} / \eta=\infty$. In the case of the dip-slip fault, the extent of viscoelastic deformation is affected by the difference in viscosity ratio. In the model with $\eta^{\prime} / \eta \geq 10$, remarkable uplift appears in the region of $y / H \geq 0.4$, and the subsidence area above the fault plane is limited. The maximum subsidence for 


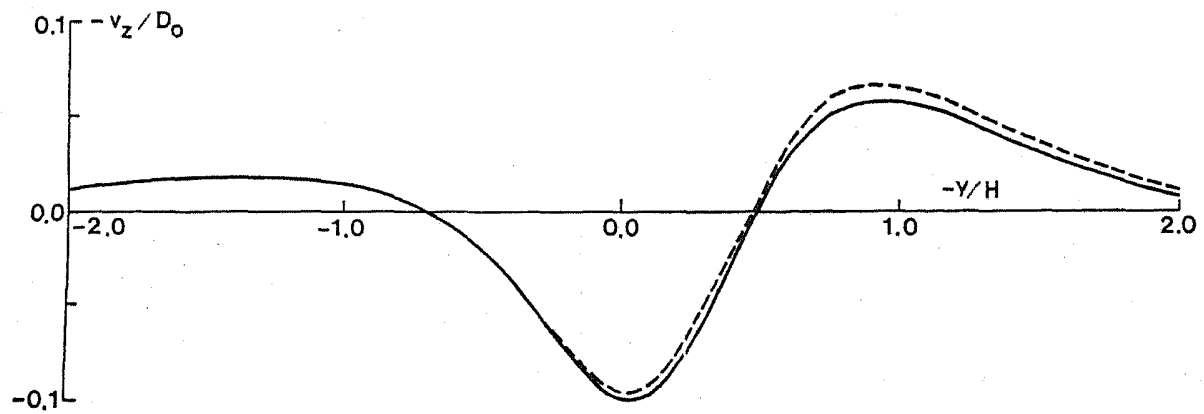

(a)

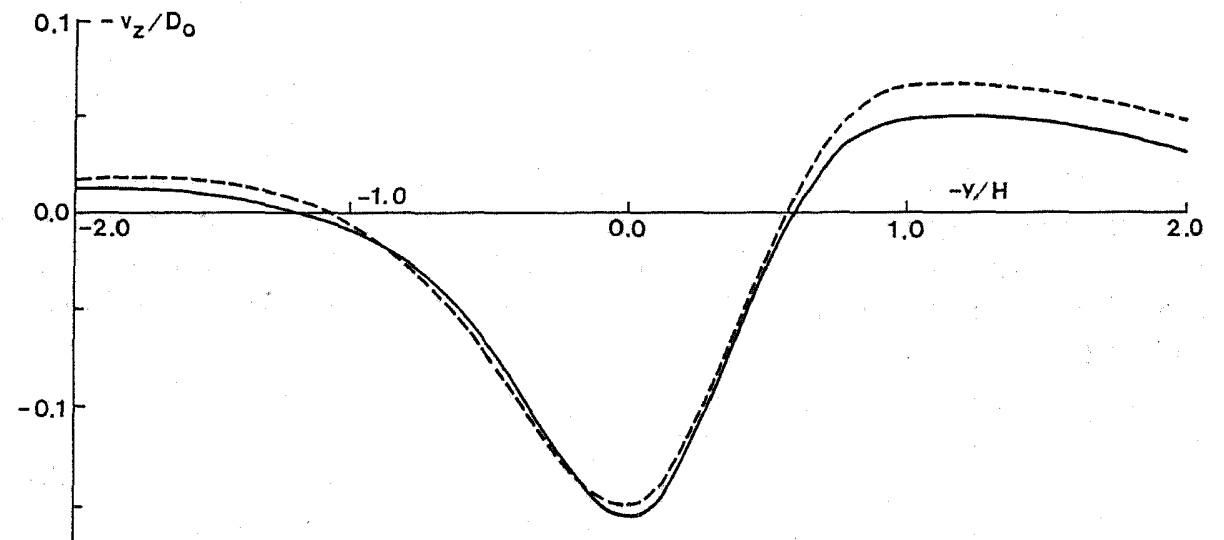

(b)

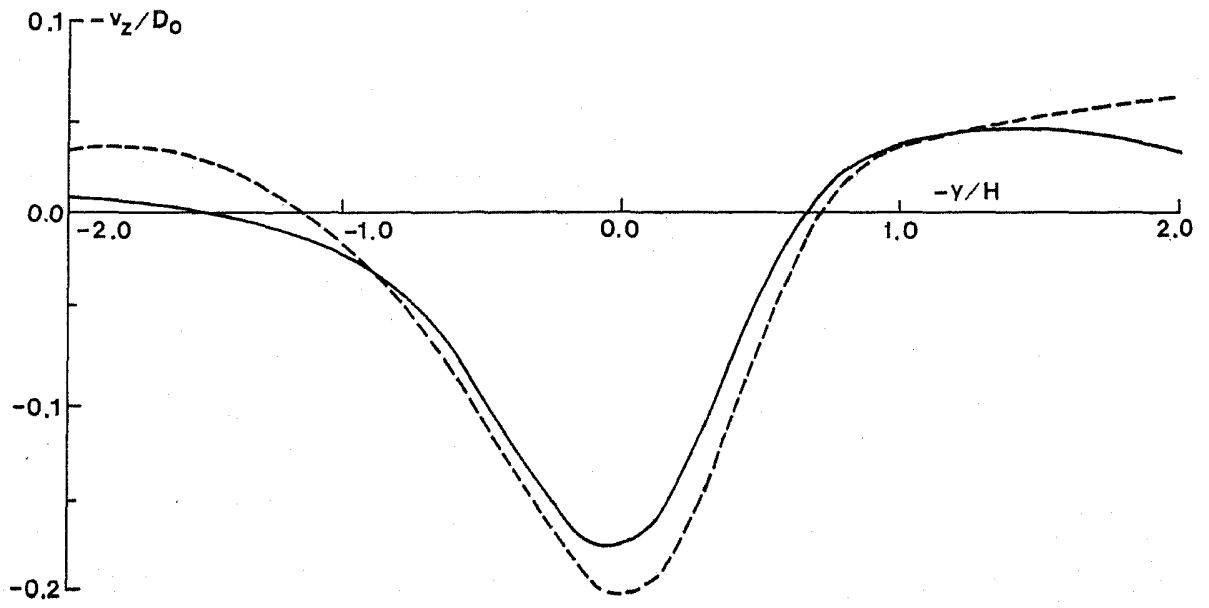

(c)

Fig. 8. Displacement profiles for gravitating case (solid curve) and non-gravitating case (dashed curve). Vertical displacements $\left(-v_{z} / D_{0}\right)$ are computed for a three-layered model with $H_{\mathrm{v}}=120 \mathrm{~km}$ and $\eta^{\prime} / \eta=10$. The fault parameters are taken to be the same as those in Fig. 7 (b). (a) $t=10 \tau$. (b) $t=50 \tau$. (c) $t=100 \tau$. 
$\eta^{\prime} / \eta=1$ is about two times greater than that for $\eta^{\prime} / \eta=\infty$.

\subsection{Effect of gravity}

The effect of gravity on the viscoelastic deformation is examined for a threelayered model with $H_{\mathrm{v}}=120 \mathrm{~km}$ and $r_{i}^{\prime} / \eta=10$.

Figure 8 shows the profiles of vertical displacements at $t=10 \tau, 50 \tau$, and $100 \tau$ due to a pure dip-slip fault, where the solid and dashed curves correspond to the gravitating and non-gravitating cases, respectively. The fault parameters used in the computation are the same as those in Fig. 7(b).

From Fig. 8, it is found that the effect of gravity becomes notable with increase of time. At the time of $10 \tau$, the displacement profile for the gravitating case is slightly different from that for the non-gravitating case in the lower-block side of the fault. In the profiles at $t=100 \tau$, it can be seen that the gravity gives a significant effect to the amount and extent of viscoelastic deformation. The maximum subsidence for the gravitating case is about $15 \%$ smaller than that for the non-gravitating case. This is in contrast with the cases of $t=10 \tau$ and $50 \tau$. RUNDLE (1982), assuming a two-layered model composed of an elastic surface layer and a viscoelastic substratum, investigated the gravity effect on the quasistatic deformations due to a pure dip-slip fault. The gross features of the profiles in Fig. 8 are consistent with those by RuNDLE (1982). In his result, however, the gravity effect is more remarkable in comparison with the present investigation. Such a difference comes from the structure model adopted for the computation. Generally, the gravity effect is notable in a structure model with low viscous layers.

In Fig. 9, vertical displacements due to the same fault at two points, $\mathrm{A}(0.0$,

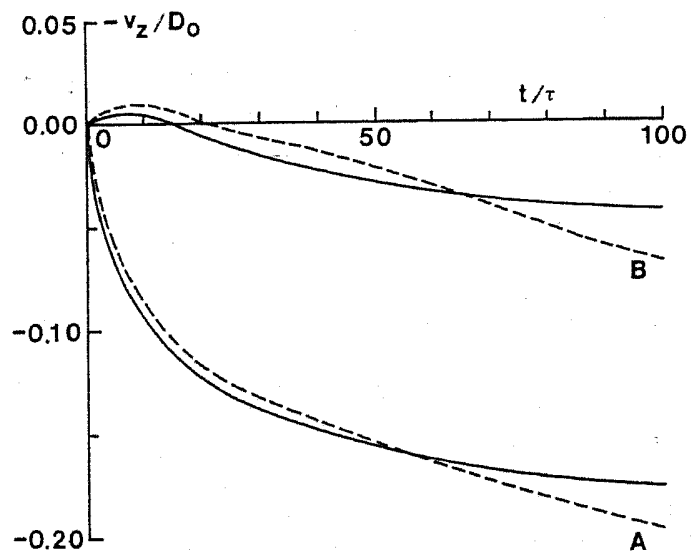

Fig. 9. Vertical displacements due to a pure dip-slip fault at two points, $A(0.0,0.0)$ and $\mathrm{B}(0.0,-0.5)$. The solid and dashed curves correspond to the gravitating case and non-gravitating case, respectively. The coordinates, structure model, and fault parameters are taken to be the same as those in Fig. 8. 
$0.0)$ and $B(0.0,-0.5)$, are illustrated with respect to time. The solid and dashed curves in this figure correspond to the gravitating and non-gravitating cases, respectively. It is found that the time-dependent behavior of viscoelastic deformation is affected notably by the gravity. The deformation for the gravitating case reaches almost a final steady state at $t=100 \tau$, which is in contrast with the nongravitating case. In the gravitating case, the stress relaxation progresses fast, and the apparent relaxation time is short in comparison with the non-gravitating case.

\section{Conclusions}

Transient crustal movements following a large earthquake are one of the interesting phenomena related with the stress relaxation in the viscoelastic region of the earth's mantle. It is widely accepted that the crust and upper mantle of the earth are characterized by three layers with different rheology, that is, the elastic surface layer (lithosphere), an intervening low viscosity layer (asthenosphere), and a substratum with relatively high viscosity. According to analyses of postglacial uplift data; the average viscosity and thickness of the low viscosity layer are estimated as $10^{20}-10^{21}$ poises and $100-200 \mathrm{~km}$, respectively. The viscosity of the substratum is 10-100 times greater than that of the low viscosity layer (MCCONELL, 1968; WalcotT, 1973; Cathles, 1975; Iwasaki and Matsu'URA, 1982).

In the present study, we modeled the uppermost part of the earth using a stratified half-space composed of elastic layers and an intervening low viscosity layer overlying a substratum with relatively high viscosity, and investigated quasistatic deformation due to a dislocation source. The rheologies of the viscous layers were assumed to be elastic dilatational and Maxwell deviatoric. Integral representations of the quasi-static displacements were derived by applying a correspondence principle of linear viscoelasticity to the associated elastic solutions. The effect of gravity was taken into account by modifying the boundary conditions with respect to normal stress.

The quasi-static deformation consists of an instantaneous elastic response at the time of faulting and a transient viscoelastic movement after the event. The viscoelastic part increases gradually with time and tends to a final steady state. We presented several numerical examples of viscoelastic displacements for a structure model of a three-layered type, which consists of an elastic surface layer, an intervening low viscosity layer, and a viscoelastic substratum with relatively high viscosity. The dislocation source was supposed to be located in the elastic surface layer.

The stress relaxation effect of the substratum on the viscoelastic surface deformation was investigated from various aspects. Displacement profiles due to a point dislocation source were presented for several three-layered models with different viscosity contrast. In a model with a low viscous substratum, the amount of viscoelastic displacement is large, and the deformations spreads in a broad area. 
In general, the stress relaxation in the substratum controls the viscoelastic deformations with long wavelength. This effect is not negligible in treating quasistatic crustal movements with a relatively long time scale. With increase of the thickness of the intervening layer, the stress relaxation in the substratum becomes less important.

The stress relaxation effect of the substratum was also examined in the case of a dimensional fault. We presented profiles of viscoelastic displacements due to a strike-slip and a dip-slip faults for structure models with different viscosity contrast. The amount and extent of the deformation strongly depend on the viscosity of the substratum. Especially, in the case of the dip-slip fault, the displacement profile in the upper-block side of the fault is affected notably by the stress relaxation in the substratum.

The effect of gravity was investigated for a dip-slip fault. We compared the vertical displacement field for the gravitating case with that for the non-gravitating case. The amount and extent of viscoelastic deformation are controlled by the gravity. Such an effect of gravity becomes notable with increase of time. In the gravitating case, the stress relaxation progresses fast, and the apparent relaxation time of deformation is short in comparison with the non-gravitating case. In the later stage of this relaxation process, the amount of viscoelastic deformation is much smaller than that for the non-gravitating case.

The author is indebted to Dr. Mitsuhiro Matsu'ura and Professor Ryosuke Sato of the Geophysical Institute, Faculty of Science, the University of Tokyo, for offering many useful suggestions to complete this work. The computations were made on HITAC 8700/8800 and HITAC $\mathrm{M} 200 \mathrm{H}$ at the Computer Centre, the University of Tokyo.

\section{APPENDIX}

In Section 2 in the text, the viscoelastic kernel functions, $V_{r(z), j}(j=1, \ldots, 4)$ and $V_{\varphi, j}(j=1,2)$ are written as

$$
\left.\begin{array}{rl}
V_{r(z), j}(t, \xi) & =-\sum_{i=1}^{7} \frac{C_{r(z), j}^{(i)}(\xi)}{\zeta_{i}(\xi)}\left(1-\mathrm{e}^{\zeta_{i(\xi) t / \tau}}\right), \\
V_{\varphi, j}(t, \xi) & =-\sum_{i=1}^{2} \frac{C_{\varphi, j}^{(i)}(\xi)}{\zeta_{i}^{\prime}(\xi)}\left(1-\mathrm{e}^{\zeta_{i}^{\prime}(\xi) t / \tau}\right) .
\end{array}\right\}
$$

The derivation of $V_{r(\varphi, z), j}$ is quite similar to those in Papers I and II. For this reason, we present only final expressions of the coefficients, $C_{r(z), j}^{(i)}(\xi)$ and $C_{\varphi, j}^{(i)}(\xi)$.

\section{Expressions of $C_{r(z), j}^{(i)}(\xi)(j=1, \ldots, 4)$}

$C_{r(z), j}^{(i)}(\xi)$ is represented as

$$
C_{r(z), j}^{(i)}(\xi)=X_{r(z), j}(\zeta, \xi) /\left.\frac{\partial}{\partial \zeta} X_{\circ}(\zeta, \xi)\right|_{\zeta=\zeta_{i}(\xi)} .
$$


Here, $X_{0}(\zeta, \xi)$ and $X_{r(z), j}(\zeta, \xi)$ are polynomials in $\zeta$, which are given by

$$
\begin{aligned}
X_{0}(\zeta, \xi) & =\sum_{i=0}^{7} \frac{f_{0}^{(i)}(\xi)}{f_{0}^{(7)}(\xi)} \zeta^{i}, \\
X_{r(z), j}(\zeta, \xi) & =\frac{f_{r(z), j}^{(7)}}{f_{0}^{(7)}(\xi)} \sum_{i=0}^{6}\left\{\frac{f_{r(z), j}^{(i)}(\xi)}{f_{r(z), j}^{(7)}(\xi)}-\frac{f_{0}^{(i)}(\xi)}{f_{0}^{(7)}(\xi)}\right\} \zeta^{i},
\end{aligned}
$$

and $\zeta_{i}(\xi)(i=1, \ldots, 7)$ are negative roots for an algebraic equation, $X_{\mathrm{o}}(\zeta, \xi)=0$. The coefficients $f_{o}^{(i)}$ and $f_{r(x), j}^{(i)}$ are defined as follows:

\subsection{Definitions of $f_{0}^{(i)}$}

The coefficients, $f_{o}^{(i)}(i=0, \ldots, 7)$ are given by

$$
\begin{aligned}
& f_{\circ}^{(0)}=g_{\circ}^{(0)} / q \gamma_{n+1}, \\
& f_{\circ}^{(i)}=\left(g_{\circ}^{(i)}-f_{\circ}^{(i-1)}\right) / q \gamma_{n+1} \quad(i=1, \ldots, 7),
\end{aligned}
$$

with

$$
g_{0}^{(i)}=N_{12}^{(i)}+N_{21}^{(i)}-N_{34}^{(i)}-N_{43}^{(i)}
$$

and

$$
q=\eta_{n} \mu_{n+1} / \eta_{n+1} \mu_{n}, \quad \gamma_{n+1}=\left(\lambda_{n+1}+\frac{2}{3} \mu_{n+1}\right) /\left(\lambda_{n+1}+2 \mu_{n+1}\right),
$$

where $N_{k l}^{(i)}$ is defined by Eq. (A.II-4) in Paper II.

\subsection{Definitions of $f_{r(z), j}^{(i)}(j=1, \ldots, 4)$}

The coefficients $f_{r(z), j}^{(i)}(i=0, \ldots, 7 ; j=1, \ldots, 4)$ are expressed as

$$
\left.\begin{array}{l}
f_{r(z), 1}^{(i)}=\left\{A_{r(z), 1}^{(i)}+A_{r(z), 2}^{(i)}+A_{r(z), 3}^{(i)}+A_{r(z), 4}^{(i)}\right\} \exp \left(-\sum_{j=1}^{m-1} \beta_{j}\right) / \prod_{j=1}^{m} C_{j}, \\
f_{r(z), 2}^{(i)}=\left\{-A_{r(z), 1}^{(i)}+A_{r(z), 2}^{(i)}+A_{r(z), 3}^{(i)}-A_{r(z), 4}^{(i)}\right\} \exp \left(-\sum_{j=1}^{m} \beta_{j}\right) / \prod_{j=1}^{m} C_{j}, \\
f_{r(z), 3}^{(i)}=\left\{\left(2-\alpha_{m}\right) A_{r(z), 2}^{(i)}+\left(1-\alpha_{m}\right) A_{r(z), 3}^{(i)}+A_{r(z), 4}^{(i)}\right\} \exp \left(-\sum_{j=1}^{m-1} \beta_{j}\right) / \prod_{j=1}^{m} C_{j}, \\
f_{r(z), 4}^{(i)}=\left\{\left(2-\alpha_{m}\right) A_{r(z), 2}^{(i)}+\left(1-\alpha_{m}\right) A_{r(z), 3}^{(i)}-A_{r(z), 4}^{(i)}\right\} \exp \left(-\sum_{j=1}^{m} \beta_{j}\right) / \prod_{j=1}^{m} C_{j},
\end{array}\right\}
$$

with

$$
\begin{aligned}
& A_{r(z), j}^{(0)}=g_{r(z), j}^{(n)} / q \gamma_{n+1}, \\
& A_{r(z), j}^{(i)}=\left(g_{r(z), j}^{(i)}-A_{r(z), j}^{(i-1)}\right) / q \gamma_{n+1} \quad(i=1, \ldots, 7),
\end{aligned}
$$

and

$$
\beta_{j}=\xi H_{j}, \quad C_{j}=\frac{1}{2}\left(1+\mathrm{e}^{-2 \beta_{j}}\right),
$$

where 


$$
\begin{aligned}
& g_{r, j}^{(i)}=N_{25}^{(i)}+N_{52}^{(i)}-N_{36}^{(i)}-N_{63}^{(i)}, \\
& g_{z, j}^{(i)}=N_{16}^{(i)}+N_{61}^{(i)}-N_{45}^{(i)}-N_{54}^{(i)} .
\end{aligned}
$$

In Eq. (A.10), $N_{k l}^{i i}$ for a certain number, $j$, is obtained from Eq. (A.II-4) in Paper II by putting

$$
R_{5}^{(i)}=Q_{1 j}^{(i)}+Q_{2 j}^{(i)}, \quad R_{6}^{(i)}=Q_{3 j}^{(i)}+Q_{4 j}^{(i)} \quad(i=0, \ldots, 4) .
$$

$Q_{k l}^{(i)}$ is the $k l$ element of a $4 \times 4$ matrix $Q^{(i)}$ which is given by the following matrix product,

where

$$
\boldsymbol{Q}^{(i)}=\boldsymbol{H}^{(i)} \cdot \boldsymbol{F}_{n-1} \cdots \boldsymbol{F}_{m+1} \cdot \boldsymbol{D}_{m}
$$

with

$$
D_{m}=\left[\begin{array}{cccc}
1 & 0 & 0 & 0 \\
0 & 1 & 0 & 0 \\
0 & 0 & \delta_{m} & 0 \\
0 & 0 & 0 & \delta_{m}
\end{array}\right]
$$

$$
\delta_{m}=\mu_{m} / \mu_{m+1},
$$

and the other matrices are defined by Eq. (A. I-11) in Paper II.

2. Expressions of $C_{\varphi, j}^{\{i\rangle}(\xi)(j=1,2)$

$C_{\varphi, j}^{(i)}(\xi)$ is represented as

$$
C_{\varphi, j}^{i i)}(\xi)=Y_{\varphi, j}(\zeta, \xi) /\left.\frac{\partial}{\partial \zeta} Y_{\circ}(\zeta, \xi)\right|_{\zeta=\zeta_{i}^{\prime}(\xi)},
$$

where, $Y_{0}(\zeta, \xi)$ and $Y_{\varphi, j}(\zeta, \xi)$ are polynomials in $\zeta$, which are given by

$$
\left.\begin{array}{rl}
Y_{\circ}(\zeta, \xi) & =\sum_{i=0}^{2} \frac{h_{\circ}^{(i)}(\xi)}{h_{\circ}^{(2)}(\xi)} \zeta^{i}, \\
Y_{\varphi, j}(\zeta, \xi) & =\frac{h_{\varphi, j}^{(2)}(\xi)}{h_{\circ}^{(2)}(\xi)} \sum_{i=0}^{1}\left\{\frac{h_{\varphi, j}^{(i)}(\xi)}{h_{\varphi, j}^{(2)}(\xi)}-\frac{h_{\circ}^{(i)}(\xi)}{h_{\circ}^{(2)}(\xi)}\right\} \zeta^{i},
\end{array}\right\}
$$

and $\zeta_{i}^{\prime}(\xi)(i=1,2)$ are negative roots for an algebraic equation, $Y_{\circ}(\zeta, \xi)=0$. The coefficients, $h_{\circ}^{(i)}$ and $h_{\varphi, j}^{(i)}$ are expressed as

and

$$
\begin{aligned}
& h_{\circ}^{(0)}=P_{11}^{\prime(1)}+P_{21}^{\prime(1)}, \\
& h_{0}^{(1)}=P_{11}^{(0)}+P_{21}^{\prime(0)}+P_{11}^{\prime(1)}+P_{21}^{\prime(1)}+P_{11}^{(2)}+P_{21}^{\prime(2)}, \\
& h_{0}^{(2)}=P_{11}^{\prime(1)}+P_{21}^{\prime(0)},
\end{aligned}
$$

with

$$
\begin{aligned}
& h_{\varphi, 1}^{(i)}=\left\{B_{\varphi, 1}^{(i)}+B_{\varphi, 2}^{(i)}\right\} \exp \left(-\sum_{j=1}^{m-1} \beta_{j}\right) / \prod_{j=1}^{m} C_{j}, \\
& h_{\varphi, 2}^{(i)}=\left\{B_{\varphi, 1}^{(i)}-B_{\varphi, 2}^{(i)}\right\} \exp \left(-\sum_{j=1}^{m} \beta_{j}\right) / \prod_{j=1}^{m} C_{j},
\end{aligned}
$$




$$
\begin{aligned}
& B_{\varphi, j}^{(0)}=Q_{1 j}^{(1)}+Q_{2 j}^{\prime(1)}, \\
& B_{\varphi, j}^{(1)}=Q_{1 j}^{\prime(0)}+Q_{2 j}^{(0)}+Q_{1 j}^{\prime(1)}+Q_{2 j}^{\prime(1)}+Q_{1 j}^{(2)}+Q_{2 j}^{(2)}, \\
& B_{\varphi, j}^{(2)}=Q_{1 j}^{\prime(0)}+Q_{2 j}^{\prime(0)} .
\end{aligned}
$$

Here, $P_{k l}^{\prime(i)}$ and $Q_{k l}^{\prime(i)}$ are the $k l$ elements of $2 \times 2$ matrices, $P^{\prime(i)}$ and $Q^{\prime(i)}$, which are given by the following matrix products,

$$
\begin{array}{ll}
\boldsymbol{P}^{\prime(i)}=\boldsymbol{H}^{\prime(i)} \cdot \boldsymbol{F}_{n-1}^{\prime(i)} \cdots \boldsymbol{F}_{1}^{\prime(i)} & (i=0, \ldots, 2), \\
\boldsymbol{Q}^{\prime(i)}=\boldsymbol{H}^{\prime(i)} \cdot \boldsymbol{F}_{n-1}^{\prime(i)} \cdots \boldsymbol{F}_{m+1}^{\prime(i)} \boldsymbol{D}_{m}^{\prime} & (i=0, \ldots, 2),
\end{array}
$$

with

$$
\begin{gathered}
\boldsymbol{H}^{\prime(0)}=\left[\begin{array}{cc}
1 & T_{n} \\
\delta_{n} T_{n} & \delta_{n}
\end{array}\right], \quad \boldsymbol{H}^{\prime(1)}=\left[\begin{array}{cc}
0 & T_{n} \\
0 & q \delta_{n}
\end{array}\right], \quad \boldsymbol{H}^{\prime(2)}=(q-1)\left[\begin{array}{cc}
0 & 0 \\
\delta_{n} T_{n} & 0
\end{array}\right] \\
\boldsymbol{F}_{j}^{\prime}=\left[\begin{array}{cc}
1 & T_{j} \\
\delta_{j} T_{j} & \delta_{j}
\end{array}\right], \quad \boldsymbol{D}_{m}^{\prime}=\left[\begin{array}{cc}
1 & 0 \\
0 & \delta_{m}
\end{array}\right]
\end{gathered}
$$

and

$$
\delta_{j}=\mu_{j} / \mu_{j+1}, \quad T_{j}=\tanh \beta_{j} .
$$

\section{REFERENCES}

Cathles, L. M., The Viscosity of the Earth's Mantle, Princeton University Press, Princeton, New Jersey, 1975.

Cohen, S. C., Postseismic surface deformations due to lithospheric and asthenospheric viscosity, Geophy. Res. Let., 6, 129-131, 1979.

Cohen, S. C., Postseismic viscoelastic surface deformation and stress. 1. Theoretical consideration, displacement and strain calculations, J. Geophys. Res., 85, 3131-3150, 1980a.

CoHen, S. C., Postseismic viscoelastic surface deformation and stress. 2. Stress theory and computation, dependence of displacement, strain and stress on fault parameters, J. Geophys. Res., 85, 3151-3158, 1980 b.

IWASAKI, T. and M. MATSU'URA, Quasi-static strains and tilts due to faulting in a layered halfspace with an intervenient viscoelastic layer, $J$. Phys. Earth, 29, 499-518, 1981.

IWASAKI, T. and M. MATSU'URA, Quasi-static crustal deformations due to a surface load: Rheological structure of the earth's crust and uppermantle, J. Phys. Earth, 30, 469-508, 1982.

Matsu'Ura, M., T. TANIMOTo, and T. IwASAKr, Quasi-static displacements due to faulting in a layered half-space with an intervenient viscoelastic layer, J. Phys. Earth, 29, 23-54, 1981.

McConell, R. K., Isostatic adjustment in a layered earth, J. Geophys. Res., 70, 5171-5188, 1965.

MCConell, R. K., Viscosity of the mantle from relaxation spectra of the isostatic adjustment, $J$. Geophys. Res., 73, 7089-7105, 1968.

Nur, A. and G. MAVKo, Postseismic viscoelastic rebound, Science, 183, 204-206, 1974.

RuNDle, J. B., Viscoelastic crustal deformation by finite quasi-static sources, J. Geophys. Res., 83, 5937-5945, 1978.

RuNDLE, J. B., Viscoelastic-gravitational deformation by a rectangular thrust fault in a layered earth, J. Geophys. Res., 87, 7787-7796, 1982.

RunDLE, J. B. and D. D. JACKSON, A three-dimensional viscoelastic model of a strike slip fault, Geophys. J. R. Astron. Soc., 49, 575-591, 1977.

SATO, R. and M. MATSU'URA, Static deformations due to the fault spreading over several layers in a multi-layered medium. Part I. Displacement, J. Phys. Earth, 21, 227-249, 1973.

WALCOTT, R. I., Structure of the earth from glacio-isostatic rebound, in Annual Reviews of the Earth and Planetary Science, ed. F. A. Donath, pp. 15-37, Annual Reviews Inc., Palo Alto, 1973. 